\title{
Neuropathic Pain in Animal Models of Nervous System Autoimmune Diseases
}

\author{
David H. Tian, Chamini J. Perera, and Gila Moalem-Taylor \\ School of Medical Sciences, University of New South Wales, Sydney, NSW 2052, Australia \\ Correspondence should be addressed to Gila Moalem-Taylor; gila@unsw.edu.au
}

Received 5 March 2013; Accepted 9 April 2013

Academic Editor: Anna Maria Aloisi

Copyright (C) 2013 David H. Tian et al. This is an open access article distributed under the Creative Commons Attribution License, which permits unrestricted use, distribution, and reproduction in any medium, provided the original work is properly cited.

\begin{abstract}
Neuropathic pain is a frequent chronic presentation in autoimmune diseases of the nervous system, such as multiple sclerosis (MS) and Guillain-Barre syndrome (GBS), causing significant individual disablement and suffering. Animal models of experimental autoimmune encephalomyelitis (EAE) and experimental autoimmune neuritis (EAN) mimic many aspects of MS and GBS, respectively, and are well suited to study the pathophysiology of these autoimmune diseases. However, while much attention has been devoted to curative options, research into neuropathic pain mechanisms and relief has been somewhat lacking. Recent studies have demonstrated a variety of sensory abnormalities in different EAE and EAN models, which enable investigations of behavioural changes, underlying mechanisms, and potential pharmacotherapies for neuropathic pain associated with these diseases. This review examines the symptoms, mechanisms, and clinical therapeutic options in these conditions and highlights the value of EAE and EAN animal models for the study of neuropathic pain in MS and GBS.
\end{abstract}

\section{Introduction}

Neuropathic pain is caused by a lesion or disease of the somatosensory nervous system either at the peripheral or central level and is a frequent presentation in a myriad of medical conditions [1]. It is characterised by abnormal sensations or hypersensitivity in the affected area, which is often combined with, or is adjacent to, areas of sensory deficit [2]. Symptoms include tactile or thermal hypoaesthesia (reduced sensation to nonpainful stimuli), hypoalgesia (reduced sensation to painful stimuli), loss of sensation, paraesthesia (abnormal sensations such as skin crawling or tingling), paroxysmal pain (e.g., shooting, electric shocklike sensations), spontaneous ongoing pain (not induced by stimulus e.g., burning sensation), and evoked pain (i.e., stimulus-induced pain), the last of which includes hyperalgesia (increased sensitivity to painful stimuli) and allodynia (perception of innocuous/non-painful stimuli as painful) [2]. In particular, neuropathic pain is common in autoimmune demyelinating diseases of the nervous system, such as multiple sclerosis (MS) and Guillain-Barre syndrome (GBS), and adversely affects millions of sufferers worldwide $[3,4]$.

Thus far, several animal models have been established to mimic features of MS and GBS, so as to better enable researchers to understand the underlying pathophysiology and immune mechanisms and to investigate better therapeutic options. For example, experimental autoimmune encephalomyelitis (EAE) serves as the classic animal model of multiple sclerosis, whereas experimental autoimmune neuritis (EAN) mimics acute inflammatory demyelinating polyneuropathy, the most common subtype of GBS [5-7]. These two models are the most widely used and accepted analogues of MS and GBS and provide many immunological parallels. In this review, we discuss the symptoms, mechanisms, and potential therapeutic strategies in neuropathic pain associated with EAE and EAN.

\section{Multiple Sclerosis and Experimental Autoimmune Encephalomyelitis}

Multiple sclerosis is a chronic, T-cell mediated autoimmune inflammatory disease of the central nervous system (CNS) that predominantly affects the myelin sheath. It is the most common cause of acquired disability in young adults in the western world [8-10]. Among the many sensory disturbances present in MS, pain-nociceptive, neuropathic, or mixed-is a highly prevalent symptom, reported by 25 to $90 \%$ of patients 
[3, 11-17]. It negatively impacts on general health, energy and vitality, mental health, and social functioning $[17,18]$, as well as impinges on daily life $[12,14,19]$. Despite its prevalence, the specific underlying mechanisms of MS pain are still not well understood [20], although elucidation has been sought through recent studies in animal models [21-23].

Experimental autoimmune encephalomyelitis has frequently served as an animal model of MS. EAE is commonly induced in genetically susceptible animal strains by immunisation with a self-antigenic epitope of myelin, which causes characteristic breakdown of the blood-brain barrier and multifocal infiltration of activated immune cells that attack the myelin sheath [8]. The ensuing immunologic response leads to chronic neuroinflammation, demyelination, and neuronal damage in the CNS. The species-specific disease course exhibits close clinical and histopathological similarities to various forms of MS [24-26], thereby presenting EAE as a suitable model to study multiple sclerosis $[27,28]$.

\section{Symptoms of Neuropathic Pain in MS and EAE}

MS patients often experience a wide range of neuropathic pain symptoms. This includes ongoing extremity pain (characterised by constant pain in the legs and feet), trigeminal neuralgia (characterised by paroxysmal attacks of electricshock-like sensations in specific facial or intraoral areas), Lhermitte's phenomenon (characterised by a transient electrical sensation that runs down the back and is related to neck movement), and thermal and mechanical sensory abnormalities [18, 29]. As behavioural models of ongoing extremity pain and paroxysmal pain in animals are currently unavailable, most animal studies have focused on thermal and mechanical abnormalities (Table 1). EAE has thus far served as the basis for preclinical research into the mechanisms of these abnormalities. Genetic, clinical, and histopathological heterogeneities of EAE models produce different sensory and pathological changes, allowing for robust representation of the various forms of pain in MS [30].

3.1. Heat Disturbances. In multiple sclerosis, neuroinflammatory lesions in the CNS produce significant somatosensory deficits, particularly for temperature discrimination, such as paradoxical heat sensations and altered heat/cold thresholds [38-40]. Up to $58 \%$ of MS patients have reported suffering from heat sensitivities, which is a significant cause of fatigue, concentration problems, and pain [41]. Such abnormalities have been paralleled in several EAE studies.

In an early study, Duckers and associates noted 23\%$58 \%$ prolongation of reaction time to noxious heat at 10 and 18 weeks following EAE induction in Lewis rats, suggestive of chronic hypoalgesia [31]. More recently, Aicher and colleagues observed a dynamic thermal response in a chronic relapsing-remitting form of EAE induced by a myelin proteolipid protein (PLP). In both male and female SJL mice, initial thermal hypoalgesia occurred concurrent with onset of clinical symptoms, later manifesting as chronic thermal hyperalgesia of the tail. Hyperalgesia was more sustained in female mice [21], reflecting a sex-linked disease profile [42]. While the magnitude and duration of tail hyperalgesia were seen to be related to the severity of motor symptoms, thus potentially cofounding the results, it was noted that the onset of hypoalgesic nociceptive responses preceded motor dysfunctions by several days [21].

Similar studies in chronic EAE induced in C57BL/6 mice with myelin oligodendrocyte glycoprotein (MOG) also reported heat hypoalgesia in the hindpaws, developing subsequent to symptomatic onset of disease, although this was also theorised by the investigators to be affected by concurrent gross locomotor disabilities [22]. In contrast, thermal hyperalgesia in the hindpaws developed during the chronic disease phase in SJL and C57BL/6 mice immunised with PLP or MOG, respectively [30]. In a study of both acute and chronic EAE induced by myelin basic protein (MBP) in rats, comparable tail heat allodynia was reported, with the onset of thermal abnormalities appearing prior to the development of clinical signs [23].

These findings show differential thermal responses and concur with case reports of heat hypoalgesia [43], as well as thermal hyperalgesia $[18,44,45]$ in MS patients.

3.2. Cold Disturbances. Cold allodynia, a reported sensory disturbance in MS patients [18], has been observed in several EAE models. In particular, cold allodynia in response to application of acetone to the hindpaws has been demonstrated in mice with a MOG-induced chronic-relapsing EAE prior to and during onset of motor disturbances [22]. Similarly, cold sensitivity at the level of the hindpaws was noted in EAE rats that were tested on a cold plate, starting before and lasting during and after clinical signs [23]. In the latter investigation, cold hyperalgesia at the level of the tail was also observed, although this was only present prior to clinical onset of EAE. Thibault and colleagues also detected no significant differences in cold allodynia and hyperalgesia between both acute and chronic EAE models, suggesting that abnormalities to cold sensitivities are independent of EAE phenotype [23]. The early onset of cold allodynia parallels the observation that neuropathic pain in MS patients often precedes or is present at clinical onset [11].

3.3. Mechanical Disturbances. In addition to thermal abnormalities, MS patients often experience tactile allodynia [11, 18, 46]. For example, both tactile hypoesthesia (reduced sensation to touch) and allodynia have been reported in relapsing remitting forms of MS $[45,47,48]$. A recent study reported high prevalence of hypoesthesia and hyperesthesia (61\% and 34\%) in patients with MS and central neuropathic pain, although this was similar to a control group of MS patients with painless sensory symptoms [40].

In chronic relapsing EAE models (using $\mathrm{MOG}_{35-55}$ in mice), robust mechanical allodynia became apparent prior to clinical signs [22], although the response times to tactile stimuli increased during disease peak (hypoalgesia), and reduced following partial amelioration of motor dysfunction. Again, this suggests confounding influence of mechanical paralysis. Similar studies using the same encephalitogenic 


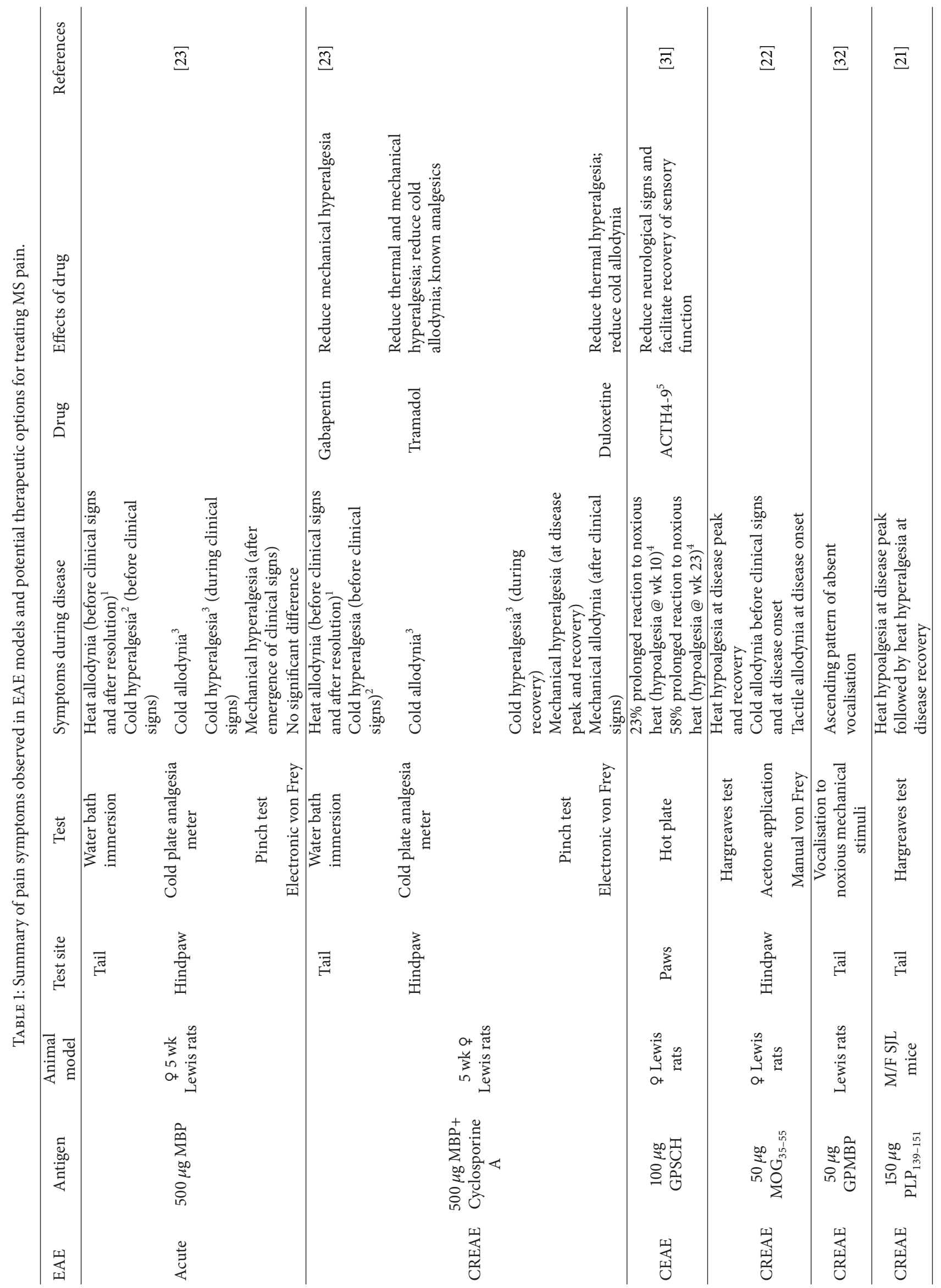




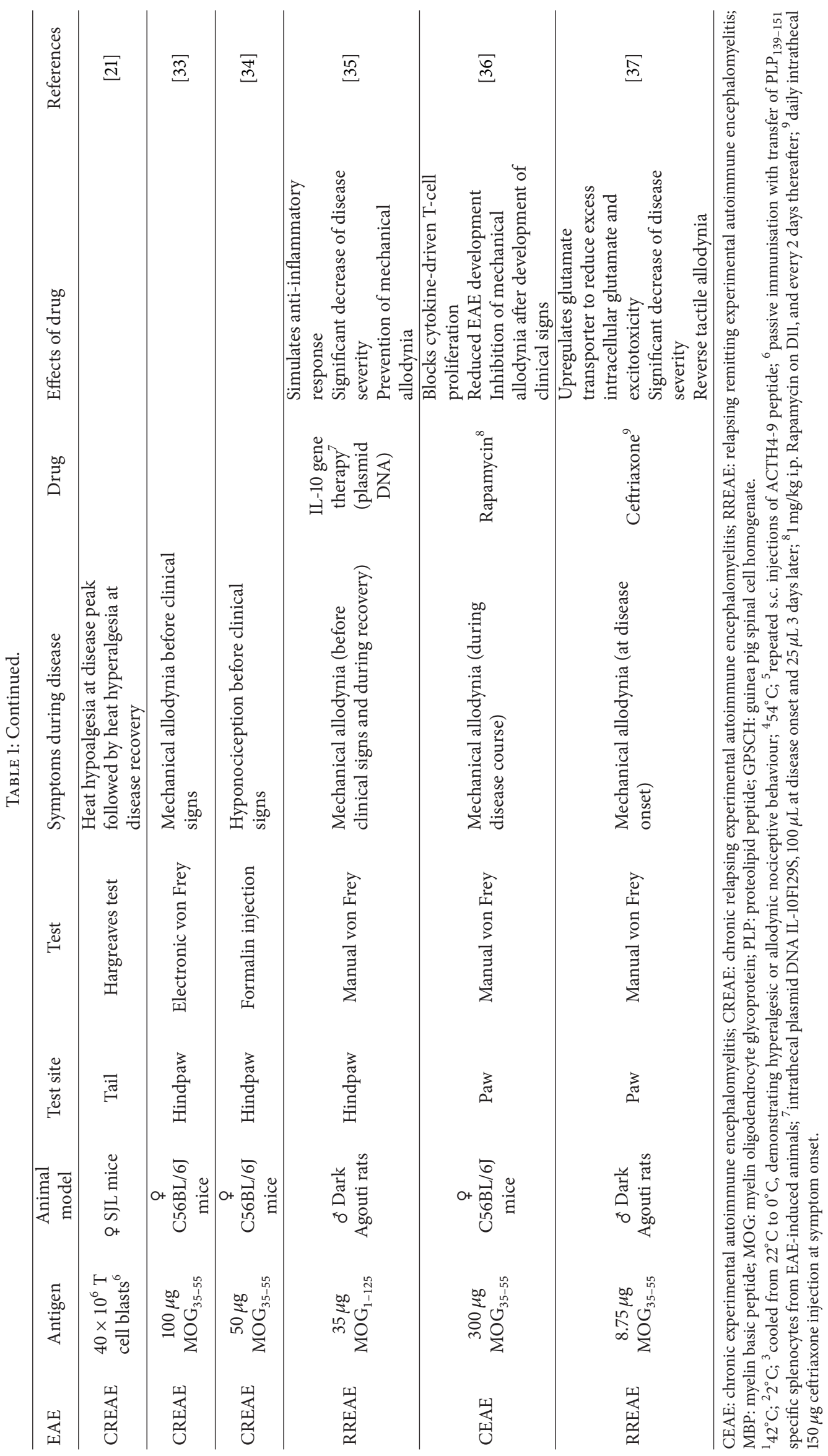


antigen $\left(\mathrm{MOG}_{35-55}\right)$ elicited comparable results of hypernociception $[33,36]$, although without hypoalgesia at disease peak. A recent study has demonstrated that the development of mechanical sensitivity is dependent upon the EAE model used; whereas SJL mice immunised with MOG developed marked mechanical allodynia during the chronic phase of the disease, C57BL/6 mice immunised with PLP developed only minor mechanical allodynia during disease onset and peak phases [30].

The robust nociceptive changes were similarly observed in a study using a rat model of MOG-induced EAE, showing periods of both decreased sensitivity to touch prior to the onset of hindlimb paralysis and increased sensitivity to touch (mechanical allodynia) during symptomatic remission [35]. Interestingly, a study using 2 doses of MOG in rats established that a $12.5 \%$ reduction in the dosage of the encephalitogenic peptide was sufficient to significantly ameliorate motor deficit profiles but did not significantly alter the robust pain states, thereby highlighting the partial independence of evoked pain presentations to motor dysfunctions [37]. This is a concept previously established through a novel study, whereby the investigator observed the absence of vocalised pain response despite noxious mechanical stimulation of the paralysed tail [32]. As vocalisation reflex can occur unhindered by tail paralysis, it can be surmised that motor paralysis (in this case, of the tail) is not the sole cause of diminished pain behaviours.

\section{Potential Mechanisms of Neuropathic Pain in MS and EAE}

As the importance of pain as a functional disability of multiple sclerosis has only recently been recognised, a clear understanding of its pathogenesis is still absent. Several theories exist to explain its mechanism, including lesions of CNS areas that process pain information, generation of enhanced response to painful stimuli due to loss of descending inhibitory nociceptive pathways, damage to somatosensory nerves, and inflammation of the spinal cord $[10,21,29$, 49].

Some of the proposed mechanisms of neuropathic pain in MS patients include thalamic or cortical deafferentation due to multiple lesions along the spinothalamocortical pathways generating ongoing extremity pain, high-frequency ectopic discharges due to demyelination of the trigeminal afferents producing symptoms of trigeminal neuralgia, and highfrequency ectopic discharges due to demyelination of the dorsal column primary afferents causing Lhermitte's phenomenon [10]. While these mechanisms have not yet been validated through animal studies, preclinical studies suggest that inflammation and gliosis are key mediators in changes in sensory functions (such as cold and tactile allodynia) seen in EAE.

It is well accepted that inflammatory cells and immunelike glial cells and their mediators facilitate central sensitisation and contribute to neuropathic pain symptoms [50]. Indeed, a recent study has shown that animals with EAE did not have altered expression of sensory neuropeptides but had a significant influx of CD3+ T cells and increased astrocyte and microglia/macrophage reactivity in the superficial dorsal horn of the spinal cord, an area associated with pain processing [22]. Furthermore, a significant increase in the level of tumour necrosis factor $\alpha$ (TNF) expression in the dorsal root ganglia (DRG) of EAE animals was found at disease peak [51]. A later study confirmed a correlation between the increase in TNF gene and protein expression in the DRG and spinal cord with the onset of neuropathic pain in rats with EAE [52]. Similar increases in the gene expression of cytokines interleukin (IL)- $1 \beta$ and IL- 6 in the spinal cords of EAE mice coincided with increased nociceptive sensitivity and deficits in object recognition [53]. Gene therapy with antiinflammatory IL-10 in animals with EAE improved motor and sensory function, prevented allodynia, and reduced glial activation in the lumbar spinal cord [35].

Further mechanisms have implicated the accumulation of infiltrating macrophages expressing purinergic $\mathrm{P}_{2} \mathrm{X}_{4}$ receptors $\left(\mathrm{P} 2 \mathrm{X}_{4} \mathrm{R}\right)$ in CNS lesions of EAE animals [54]. As activation of these receptors by adenosine triphosphate is implicated in the microglial response to peripheral nerve injury and neuropathic pain symptoms, an association between $\mathrm{P} 2 \mathrm{X}_{4} \mathrm{R}$ and neuropathic pain in EAE is suggested [55]. Additionally, increased phosphorylation of transcription factor cyclic AMP response element-binding protein (CREB) has also been observed at disease peak in EAE lesions, particularly in the dorsal horn sensory neurons [56], which are associated with the generation and maintenance of neuropathic pain. Similar involvement of chemokines in leukocyte recruitment, immune regulation, and T-cell polarisation is believed to significantly impact on pain regulation. For example, CCL2, a chemokine with elevated levels in MS patients, amplifies inflammatory responses in EAE [57, 58], while intrathecal administration of CCL2 chemokine is sufficient to induce mechanical allodynia in naïve animals but not in CCL2-receptor knockout mice [59-61].

Dysregulation of the glutamatergic system, caused by reduced glutamate transporter expression in spinal cords, has been implicated in abnormal pain sensitivity in mice with MOG-induced EAE. For example, EAE mice showed a lack of behavioural response to formalin stimulation, a behavioural model of injury-induced central sensitization. This hyporesponsiveness was attributed to a decreased expression of the glutamate transporters EAAT-1 and EAAT-2 in the spinal cord [34]. Furthermore, pharmacological treatment to upregulate the levels of EAAT-2 in mice with EAE resulted in prevention of tactile hypersensitivity and normalisation of performance in cognitive assays [53].

\section{Clinical Applications of EAE Neuropathic Pain Models}

EAE has proven to be a successful therapeutic preclinical model for MS. Indeed, a number of approved drugs and current phase II and III trials for MS were first examined in EAE models [62].

Several pharmacotherapies used to treat pain in multiple sclerosis have shown similar efficacies in EAE. For example, Gabapentin, a $\gamma$-aminobutyric acid (GABA) analogue used 
by up to $19 \%$ of MS sufferers $[63,64]$, is highly effective in ameliorating pain symptoms in MS, such as trigeminal neuralgia and tonic spasms [65-68]. Moreover, using Gabapentin, Thibault and colleagues demonstrated a significant reduction of mechanical hyperalgesia in EAE murine models, highlighting the effectiveness of GABA analogues and their therapeutic potentials on neuropathic pain in EAE models [23].

There also exist several promising avenues of pharmaceutical research. Lisi and associates have established that prophylactic Rapamycin administration, a macrocyclic antibiotic with immunosuppressive activity, is able to reduce disease severity and ameliorate pain behaviour in EAE animals [36], confirming similar rodent studies [69-71]. It is theorised that by regulating effector $\mathrm{T}$ cell and regulatory T-cell function [72], Rapamycin is able to modulate cytokine release, particularly interferon (IFN)- $\gamma$ [73], a potent cytokine implicated in neuropathic pain [50].

Another promising candidate for MS pain amelioration targets glutamate transporters. MS patients are known to have an elevated concentration and/or altered transport of glutamate in the CNS [74-78], partly due to glutamate released by invading $\mathrm{T}$ cells and macrophages $[79,80]$. This increases extracellular accumulation of glutamate through the downregulation of glutamate transporters and impairment of glial glutamate uptake [81]. The excess glutamate concentrations allow for prolongation of calcium-permeable ionotropic glutamate receptor activation on neural and glial cells, leading to excitotoxic CNS tissue damage $[82,83]$. Studies in EAE rodent models have demonstrated $50 \%$ reduction in glial glutamate transporter (GLT-1) spinal expression compared to normal animals $[37,84]$. In chronic EAE models, administration of ceftriaxone, a third-generation cephalosporin antibiotic which upregulates CNS glutamate transporters, has not only shown to limit and attenuate clinical symptoms $[37,85]$ but also shown to significantly reverse tactile allodynia [37] and normalise facets of cognitive functioning [53]. Normalisation of pain behaviour has been confirmed using other compounds known to promote glutamate transporter activity in EAE models, such as MS-153 [34].

As MS is a predominantly proinflammatory disease, anti-inflammatory agents predictably demonstrate significant therapeutic potential. Currently, several drugs exist that effectively target the inflammatory process in MS patients [86-89]. In EAE, lumbar intrathecal injections of a plasmid DNA with mutated IL-10 gene, designed to stimulate an anti-inflammatory response, reduced disease course and prevented mechanical allodynia [35]. Furthermore, FTY720, a sphingosine 1-phosphate receptor modulator, has been shown to suppress EAE development in several rodent models [9093] by reducing the infiltration of $\mathrm{CD} 4+\mathrm{T}$ cells, macrophages, and proinflammatory cytokines [93-96], as well as by modulating signalling pathways on glial cells $[97,98]$. In addition to confining lymphocytes to lymphoid tissue [94] and preventing and reversing pathological disturbances to pre- and postsynaptic glutamate transmission [99], FTY720 is thought to induce endogenous repair mechanisms in the CNS, as it preferentially localises to myelin sheath [100]. Clinically, FTY720 has reduced MS relapse rates and lesion frequency
[101-103]. While these studies focus on disease amelioration, Balatoni and associates have demonstrated in a chronic EAE model that prophylactic application of FTY720 prevented evoked potential disturbances of the somatosensory system [104], raising the possibility of using FTY720 to modulate neuropathic pain. In support of this, a recent study has shown that administration of FTY720 reduces mechanical and thermal allodynia in animals with neuropathic pain caused by peripheral nerve injury [105].

\section{Guillain-Barre Syndrome and Experimental Autoimmune Neuritis}

Guillain-Barre syndrome is the most common acute inflammatory demyelinating neuropathy in the peripheral nervous system (PNS), and as such can almost be considered a counterpart to multiple sclerosis. It affects 1-2 individuals per 100,000, with a greater disposition towards men [106]. GBS is a common cause of neuromuscular paralysis, characterised by areflexia or acute hyperreflexia, and can be effectively treated with immunotherapies such as intravenous immunoglobulin. However, despite immunotherapy, GBS has a $5 \%$ mortality rate, with up to $20 \%$ of patients remaining severely disabled [107]. Other symptoms of GBS include sensory impairments, such as moderate to severe nociceptive and neuropathic pain $[4,108,109]$. In fact, pain is a highly prevalent symptom, with $55-85 \%$ of sufferers complaining of paraesthesia/dysaesthesia, backache and sciatica, neck pain, muscle pain, joint pain, and visceral pain [4, 109].

Experimental autoimmune neuritis is a T-cell-mediated acute demyelinating inflammatory disease of the PNS widely used as an animal model of the acute inflammatory demyelinating polyneuropathy, the most common form of GBS [110]. First successfully induced in rabbits by Waksman and Adams in 1955, EAN is characterised by degeneration of myelin sheaths, proliferation of histiocytes, breakdown of blood-nerve barrier, and localised PNS inflammation with infiltration of lymphocytic and mononuclear cells [6].

EAN can be induced by immunisation with neuritogenic peripheral nerve myelin components, purified myelin proteins (such as P0, P2, or PMP-22), or synthetic peptides of myelin proteins [111, 112], or by passive transfer of $\mathrm{T}$ cells sensitised to these proteins. Susceptible animals (such as rats, mice, rabbits, and guinea pigs) induced with EAN develop monophasic disease characterised by weight loss, ascending progressive paralysis, and spontaneous recovery.

\section{Symptoms of Neuropathic Pain in GBS and EAN}

Neuropathic pain, primarily affecting the distal extremities, represents a common and severe symptom in patients with GBS and is more common and persistent than nonneuropathic pain [113]. Dysaesthetic extremity pain, described as burning, tingling, or shock-like sensations, has been reported in up to $49 \%$ of GBS patients [109]. GBS patients also experience altered thermal sensations, with significantly higher 
warm threshold temperatures and lower cold threshold temperatures as compared to age- and gender-matched controls [114]. In support of this, a recent study has shown that GBS patients have a significantly more severe impairment of cold detection thresholds, heat pain thresholds, and responses to suprathreshold heat stimuli in the foot, as compared to patients with nonneuropathic pain or without pain [113]. In addition, GBS patients suffer from brush-induced allodynia [113].

The thermal and tactile sensory abnormalities evident in GBS are reflected in EAN models (Table 2). Behavioural tests of pain hypersensitivity in EAN, including thermal hyperalgesia and mechanical allodynia, have frequently served as tools to study GBS sensory dysfunctions. For example, Moalem-Taylor and colleagues were able to observe significant mechanical allodynia and thermal hyperalgesia in both hindpaws and forepaws of rats with EAN [115]. A subsequent study confirmed the development of neuropathic pain in EAN animals and further demonstrated that mechanical allodynia preceded the onset of neurological signs and persisted after cessation of locomotor deficit [116].

\section{Potential Mechanisms of Neuropathic Pain in GBS and EAN}

Despite its prevalence, the mechanisms of neuropathic pain in GBS patients remain unknown. It has been suggested that in the acute phase of GBS, neuropathic pain results from nerve inflammation, whereas in the chronic phase of the disease, neuropathic pain results from degeneration of sensory nerve fibres [121]. Recently, it has been shown that a considerable reduction in intraepidermal nerve fibre density at the distal leg is evident early in the disease and correlates with pain intensity in the acute phase of GBS [122]. Furthermore, impairment of small myelinated and unmyelinated nociceptive fibres is significantly greater in GBS patients with neuropathic pain than in those without neuropathic pain. The severity of such impairment during the acute phase of GBS is predictive of chronic neuropathic pain [113].

To date, very few research laboratories have studied the mechanisms underlying neuropathic pain in EAN animals. However, existing studies have implicated several inflammatory mediators and cells in the initiation and maintenance of neuropathic pain in EAN through secretion of inflammatory mediators that sensitise nociceptors to amplify pain hypersensitivity. For example, greater numbers of $\mathrm{T}$ cells, antigen-presenting cells, and macrophages were observed in peripheral nerves of EAN animals [115]. These infiltrating leukocytes in the PNS may play a role in EAN-induced pain by releasing proinflammatory cytokines such as IL18 (an IFN- $\gamma$ inducing factor, produced by macrophages) with significantly greater IL-18 expression observed in nerve roots of EAN rats and significantly higher serum levels of IL-18 detected in GBS patients as compared to control subjects [123]. Cells immunoreactive for inducible nitric oxide synthase and TNF have been also observed in the DRGs of animals with EAN [124].
Additionally, there exists accumulating evidence that microglia become activated following PNS damage and contribute to sensitisation of central nociceptors through the production of proinflammatory cytokines, chemokines, and extracellular proteases [50]. Indeed, an increase in the number of microglial cells has been demonstrated in rats with EAN [116, 120]. In particular, the association of the time course of mechanical allodynia and spinal upregulation of $\mathrm{P} 2 \mathrm{X}_{4} \mathrm{R}$ on spinal microglia in lumbar dorsal horns in EAN rats has been successfully observed by Zhang and colleagues [116]. This suggests that activation of $\mathrm{P} 2 \mathrm{X}_{4} \mathrm{R}$ drives the release of brain-derived neurotrophic factor from spinal microglia, a cellular substrate that causes disinhibition of pain-transmitting spinal lamina I neurons and mediates aberrant nociceptive processing in the spinal cord [125]. The involvement of transmembrane chemokines such as CX3CL1 (fractalkine) has also been implicated, as it plays a key role in mediating neuron-microglia interactions in nociceptive transmission. Elevated levels of CX3CL1 have been recorded in GBS patients [126], while in EAN rats, extensive upregulation of immunoreactivity for CX3CL1 and its receptor CX3CR1 in the dorsal horn has been shown to correlate with the establishment of mechanical allodynia [120].

Taken together, development and maintenance of neuropathic pain in EAN models may result from (a) demyelination and degeneration of sensory nerve fibres, (b) autoimmune inflammation in the PNS, and (c) spinal glial activation in the CNS, therefore providing a useful model for finding novel therapeutic approaches for GBS-related pain.

\section{Clinical Applications of EAN Neuropathic Pain Models}

Although the studies on pain in EAN are inadequate to date, there are a few significant approaches that may be therapeutic in relieving pain in GBS patients.

Firstly, immunotherapeutic approaches that enhance the numbers of immunosuppressive FoxP ${ }^{+}$regulatory $\mathrm{T}$ (Treg) cells and decrease neuroinflammation have demonstrated potential. Recently, it has been shown that treatment with CD28 superagonist, a Treg cell expander, resulted in a significant amelioration of EAN severity and mechanical allodynia, with associated reduction of neuroinflammatory responses [119]. Treatment with Compound A, a plantderived ligand of glucocorticoid receptors that enhances Treg cells in blood of EAN animals, is also able to attenuate mechanical allodynia [118]. The same study further observed that Compound A reduced microglial activation and IL- $1 \beta$ and TNF upregulation in the spinal cord, increased the numbers of anti-inflammatory M2 macrophages in sciatic nerves, and modulated lymphoid cytokines to an anti-inflammatory profile [118].

Furthermore, statins, which are used to treat hypercholesterolaemia in humans, are reported to potentiate antiinflammatory and immunomodulatory effects, including deviation of helper T cell Type 1 (Th1) mediated proinflammatory response to Th2 mediated anti-inflammatory response, 


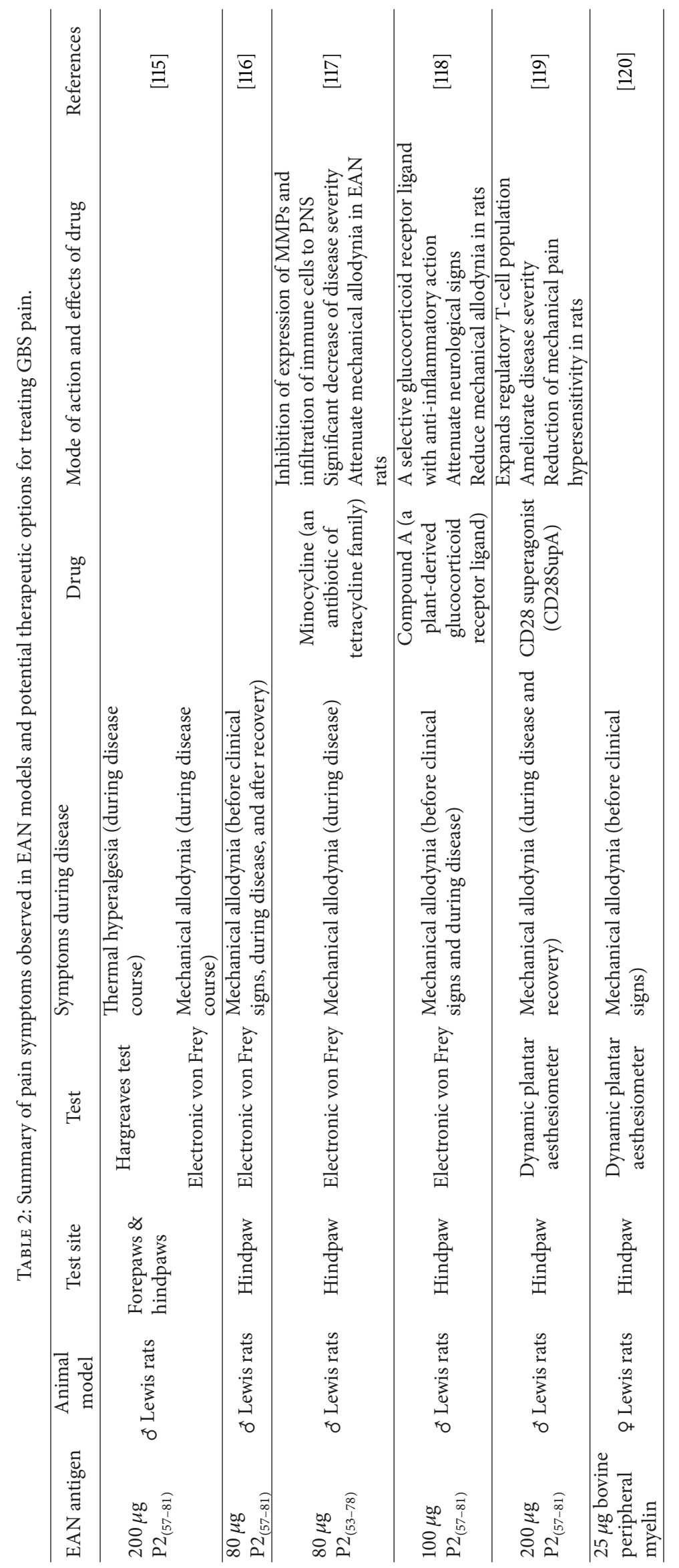


inhibition of Th1 and Th17 mediated autoimmune response, inhibition of maturation and activation of antigen presenting cells, and increasing the numbers of $\mathrm{CD} 4^{+} \mathrm{CD} 25^{+} \mathrm{FoxP} 3^{+}$ Treg cells [127]. Recent studies have found that administration of atorvastatin reduces EAN severity through a similar mechanism [127], while treatment with rosuvastatin and simvastatin prevents the development of thermal hyperalgesia and mechanical allodynia and significantly reduces spinal glial activation following peripheral nerve injury [128]. This highlights the potential for statins to manage neuropathic pain in GBS.

In addition, the existing evidence for the role of microglia in neuropathic pain suggests that controlling spinal glial activation may result in pain amelioration in GBS. In particular, inhibition of microglial activation and alleviation of mechanical allodynia has been successfully observed by peritoneal administration of minocycline, which attenuates TNF and decreases proinflammatory cytokine response $[117,129]$. Elevated levels of CX3CL1 in GBS suggest that inhibiting CX3CL1/CX3CR1 interactions will negatively affect microglial activation [120] and might prevent the development of neuropathic pain.

Other possible therapeutic approaches include inhibiting matrix metalloproteinases (MMPs). MMPs comprise a large family of proteases that have been implicated in the generation of neuroinflammation and the development of neuropathic pain through the cleavage of extracellular matrix proteins, cytokines, and chemokines [130]. MMP-9 and MMP-7 were found to be selectively upregulated during EAN and expressed in nerves of GBS patients [131]. BB-1101, a broad spectrum MMP inhibitor, has already demonstrated potential in preventing the development of EAN [130], and reduced expression of MMP-9 by treatment with minocycline was associated with improved EAN outcome and reduced mechanical allodynia [117].

\section{Summary}

Although much has been uncovered in the past few decades about the nervous system autoimmune disorders of MS and GBS, the clear pathogenesis of these diseases has not been fully elucidated. However, utilisation of animal models, in particular EAE and EAN, has significantly advanced our understanding and provided a platform for development and investigation of new therapies. Recently it has become clear that neuropathic pain is a common debilitating symptom in MS and GBS and that some of the changes in pain sensitivity observed in these patients can be mimicked in EAE and EAN animals. Tables 1 and 2 summarise neuropathic pain symptoms observed in EAE and EAN, respectively, and the therapeutic agents tested in these animal models. Many complex mechanisms are involved in mediating the various sensory changes, and we are only now beginning to understand the mechanisms underlying neuropathic pain in MS and GBS. A recent study in humans has demonstrated an autoimmune basis for some types of chronic idiopathic pain highlighting the role of autoimmune antibodies and cells in pain mediation [132]. A concerted effort is required to elicit more information regarding the mechanisms underlying neuropathic pain in MS and GBS to better enable the development of more effective treatments.

\section{Acknowledgment}

This work was supported by a grant from the National Health and Medical Research Council of Australia to G.M.T.

\section{References}

[1] R. D. Treede, T. S. Jensen, J. N. Campbell et al., "Neuropathic pain: redefinition and a grading system for clinical and research purposes," Neurology, vol. 70, no. 18, pp. 1630-1635, 2008.

[2] R. Baron, A. Binder, and G. Wasner, "Neuropathic pain: diagnosis, pathophysiological mechanisms, and treatment," The Lancet Neurology, vol. 9, no. 8, pp. 807-819, 2010.

[3] D. E. Moulin, K. M. Foley, and G. C. Ebers, "Pain syndromes in multiple sclerosis," Neurology, vol. 38, no. 12, pp. 1830-1834, 1988.

[4] B. Pentland and S. M. Donald, "Pain in the Guillain-Barre syndrome: a clinical review," Pain, vol. 59, no. 2, pp. 159-164, 1994.

[5] T. M. Rivers, D. H. Sprunt, and G. P. Berry, "Observations on attempts to produce acute disseminated encephalomyelitis in monkeys," Journal of Experimental Medicine, vol. 58, pp. 39-53, 1933.

[6] B. H. Waksman and R. D. Adams, "Allergic neuritis: an experimental disease of rabbits induced by the injection of peripheral nervous tissue and adjuvants," Journal of Experimental Medicine, vol. 102, pp. 213-236, 1955.

[7] R. Gold, H. P. Hartung, and K. V. Toyka, "Animal models for autoimmune demyelinating disorders of the nervous system," Molecular Medicine, vol. 6, no. 2, pp. 88-91, 2000.

[8] M. Sospedra and R. Martin, "Immunology of multiple sclerosis," Annual Review of Immunology, vol. 23, pp. 683-747, 2005.

[9] H. F. McFarland and R. Martin, "Multiple sclerosis: a complicated picture of autoimmunity," Nature Immunology, vol. 8, no. 9, pp. 913-919, 2007.

[10] A. Truini, P. Barbanti, C. Pozzilli, and G. Cruccu, "A mechanism-based classification of pain in multiple sclerosis," Journal of Neurology, vol. 260, pp. 351-367, 2013.

[11] A. Österberg, J. Boivie, and K. A. Thuomas, "Central pain in multiple sclerosis: prevalence and clinical characteristics," European Journal of Pain, vol. 9, no. 5, pp. 531-542, 2005.

[12] K. B. Svendsen, T. S. Jensen, K. Overvad, H. J. Hansen, N. Koch-Henriksen, and F. W. Bach, "Pain in patients with multiple sclerosis: a population-based study," Archives of Neurology, vol. 60, no. 8, pp. 1089-1094, 2003.

[13] C. J. Archibald, P. J. McGrath, P. G. Ritvo et al., "Pain prevalence, severity and impact in a clinic sample of multiple sclerosis patients," Pain, vol. 58, no. 1, pp. 89-93, 1994.

[14] D. M. Ehde, L. E. Gibbons, L. Chwastiak, C. H. Bombardier, M. D. Sullivan, and G. H. Kraft, "Chronic pain in a large community sample of persons with multiple sclerosis," Multiple Sclerosis, vol. 9, no. 6, pp. 605-611, 2003.

[15] C. Solaro, G. Brichetto, M. P. Amato et al., "The prevalence of pain in multiple sclerosis: a multicenter cross-sectional study," Neurology, vol. 63, no. 5, pp. 919-921, 2004.

[16] D. B. Clifford and J. L. Trotter, "Pain in multiple sclerosis," Archives of Neurology, vol. 41, no. 12, pp. 1270-1272, 1984. 
[17] L. V. Kalia and P. W. O'Connor, "Severity of chronic pain and its relationship to quality of life in multiple sclerosis," Multiple Sclerosis, vol. 11, no. 3, pp. 322-327, 2005.

[18] K. B. Svendsen, T. S. Jensen, H. J. Hansen, and F. W. Bach, "Sensory function and quality of life in patients with multiple sclerosis and pain," Pain, vol. 114, no. 3, pp. 473-481, 2005.

[19] A. G. Beiske, E. D. Pedersen, B. Czujko, and K. M. Myhr, "Pain and sensory complaints in multiple sclerosis," European Journal of Neurology, vol. 11, no. 7, pp. 479-482, 2004.

[20] A. B. O’Connor, S. R. Schwid, D. N. Herrmann, J. D. Markman, and R. H. Dworkin, "Pain associated with multiple sclerosis: systematic review and proposed classification," Pain, vol. 137, no. 1, pp. 96-111, 2008.

[21] S. A. Aicher, M. B. Silverman, C. W. Winkler, and B. F. Bebo Jr., "Hyperalgesia in an animal model of multiple sclerosis," Pain, vol. 110, no. 3, pp. 560-570, 2004.

[22] C. J. Olechowski, J. J. Truong, and B. J. Kerr, "Neuropathic pain behaviours in a chronic-relapsing model of experimental autoimmune encephalomyelitis (EAE)," Pain, vol. 141, no. 1-2, pp. 156-164, 2009.

[23] K. Thibault, B. Calvino, and S. Pezet, "Characterisation of sensory abnormalities observed in an animal model of multiple sclerosis: a behavioural and pharmacological study," European Journal of Pain, vol. 15, no. 3, pp. 231.e1-231.e16, 2011.

[24] R. Furlan, C. Cuomo, and G. Martino, "Animal models of multiple sclerosis," Methods in Molecular Biology, vol. 549, pp. 157-173, 2009.

[25] B. Schreiner, F. L. Heppner, and B. Becher, "Modeling multiple sclerosis in laboratory animals," Seminars in Immunopathology, vol. 31, no. 4, pp. 479-495, 2009.

[26] R. H. Swanborg, "Animal models of human disease. Experimental autoimmune encephalomyelitis in rodents as a model for human demyelinating disease," Clinical Immunology and Immunopathology, vol. 77, no. 1, pp. 4-13, 1995.

[27] C. S. Constantinescu, N. Farooqi, K. O’Brien, and B. Gran, "Experimental autoimmune encephalomyelitis (EAE) as a model for multiple sclerosis (MS)," British Journal of Pharmacology, vol. 164, pp. 1079-1106, 2011.

[28] E. Mix, H. Meyer-Rienecker, and U. K. Zettl, "Animal models of multiple sclerosis for the development and validation of novel therapies: potential and limitations," Journal of Neurology, vol. 255, supplement 6, pp. 7-14, 2008.

[29] A. Truini, F. Galeotti, S. La Cesa et al., "Mechanisms of pain in multiple sclerosis: a combined clinical and neurophysiological study," Pain, vol. 153, pp. 2048-2054, 2012.

[30] J. Lu, M. Kurejova, L. N. Wirotanseng, R. A. Linker, R. Kuner, and A. Tappe-Theodor, "Pain in experimental autoimmune encephalitis: a comparative study between different mouse models," Journal of Neuroinflammation, vol. 9, Article ID Article number233, 2012.

[31] H. J. Duckers, R. P. van Dokkum, J. Verhaagen, F. H. Lopes da Silva, and W. H. Gispen, "Functional and neurophysiological evidence of the efficacy of trophic pharmacotherapy using an adrenocorticotrophic hormone4-9 analog in experimental allergic encephalomyelitis, an animal model of multiple sclerosis," Neuroscience, vol. 71, no. 2, pp. 507-521, 1996.

[32] M. P. Pender, "Ascending impairment of nociception in rats with experimental allergic encephalomyelitis," Journal of the Neurological Sciences, vol. 75, no. 3, pp. 317-328, 1986.

[33] D. H. Rodrigues, D. Sachs, and A. L. Teixeira, "Mechanical hypernociception in experimental autoimmune encephalomyelitis," Arquivos de Neuro-Psiquiatria, vol. 67, no. 1, pp. 78-81, 2009.

[34] C. J. Olechowski, A. Parmar, B. Miller et al., "A diminished response to formalin stimulation reveals a role for the glutamate transporters in the altered pain sensitivity of mice with experimental autoimmune encephalomyelitis (EAE)," Pain, vol. 149, no. 3, pp. 565-572, 2010.

[35] E. Sloane, A. Ledeboer, W. Seibert et al., "Anti-inflammatory cytokine gene therapy decreases sensory and motor dysfunction in experimental Multiple Sclerosis: MOG-EAE behavioral and anatomical symptom treatment with cytokine gene therapy," Brain, Behavior, and Immunity, vol. 23, no. 1, pp. 92-100, 2009.

[36] L. Lisi, P. Navarra, R. Cirocchi et al., "Rapamycin reduces clinical signs and neuropathic pain in a chronic model of experimental autoimmune encephalomyelitis," Journal of Neuroimmunology, vol. 243, pp. 43-51, 2012.

[37] K. M. Ramos, M. T. Lewis, K. N. Morgan et al., "Spinal upregulation of glutamate transporter GLT-1 by ceftriaxone: therapeutic efficacy in a range of experimental nervous system disorders," Neuroscience, vol. 169, no. 4, pp. 1888-1900, 2010.

[38] D. Bowsher, "Central pain following spinal and supraspinal lesions," Spinal Cord, vol. 37, no. 4, pp. 235-238, 1999.

[39] C. Hansen, H. C. Hopf, and R. D. Treede, "Paradoxical heat sensation in patients with multiple sclerosis. Evidence for a supraspinal integration of temperature sensation," Brain, vol. 119, no. 5, pp. 1729-1736, 1996.

[40] A. Österberg and J. Boivie, "Central pain in multiple sclerosis: sensory abnormalities," European Journal of Pain, vol. 14, no. 1, pp. 104-110, 2010.

[41] G. Flensner, A. C. Ek, O. Söderhamn, and A. M. Landtblom, "Sensitivity to heat in MS patients: a factor strongly influencing symptomology: an explorative survey," BMC Neurology, vol. 11, article 27, 2011.

[42] B. F. Bebo Jr., J. C. Schuster, A. A. Vandenbark, and H. Offner, "Gender differences in experimental autoimmune encephalomyelitis develop during the induction of the immune response to encephalitogenic peptides," Journal of Neuroscience Research, vol. 52, no. 4, pp. 420-426, 1998.

[43] A. R. Burkey and S. Abla-Yao, "Successful treatment of central pain in a multiple sclerosis patient with epidural stimulation of the dorsal root entry zone," Pain Medicine, vol. 11, no. 1, pp. 127132,2010

[44] M. G. Grasso, A. Clemenzi, A. Tonini et al., "Pain in multiple sclerosis: a clinical and instrumental approach," Multiple Sclerosis, vol. 14, pp. 506-513, 2008.

[45] C. Morin, M. C. Bushnell, M. B. Luskin, and A. D. Craig, "Disruption of thermal perception in a multiple sclerosis patient with central pain," Clinical Journal of Pain, vol. 18, no. 3, pp. 191195, 2002.

[46] G. Cruccu, A. Biasiotta, S. Di Rezze et al., "Trigeminal neuralgia and pain related to multiple sclerosis," Pain, vol. 143, no. 3, pp. 186-191, 2009.

[47] L. De Santi, L. Monti, E. Menci, M. Bellini, and P. Annunziata, "Clinical-radiologic heterogeneity of occipital neuralgiform pain as multiple sclerosis relapse," Headache, vol. 49, no. 2, pp. 304-307, 2009.

[48] T. Sakai, S. Tomiyasu, T. Ono, H. Yamada, and K. Sumikawa, "Multiple sclerosis with severe pain and allodynia alleviated by oral ketamine," Clinical Journal of Pain, vol. 20, no. 5, pp. 375376, 2004. 
[49] G. Moalem and D. J. Tracey, "Immune and inflammatory mechanisms in neuropathic pain," Brain Research Reviews, vol. 51, no. 2, pp. 240-264, 2006.

[50] P. J. Austin and G. Moalem-Taylor, "The neuro-immune balance in neuropathic pain: involvement of inflammatory immune cells, immune-like glial cells and cytokines," Journal of Neuroimmunology, vol. 229, no. 1-2, pp. 26-50, 2010.

[51] M. Melanson, P. Miao, D. Eisenstat et al., "Experimental autoimmune encephalomyelitis-induced upregulation of tumor necrosis factor-alpha in the dorsal root ganglia," Multiple Sclerosis, vol. 15, no. 10, pp. 1135-1145, 2009.

[52] F. Begum, W. Zhu, C. Cortes, B. MacNeil, and M. Namaka, "Elevation of tumor necrosis factor alpha in dorsal root ganglia and spinal cord is associated with neuroimmune modulation of pain in an animal model of multiple sclerosis," Journal of Neuroimmune Pharmacology, 2013.

[53] C. J. Olechowski, G. Tenorio, Y. Sauve, and B. J. Kerr, "Changes in nociceptive sensitivity and object recognition in experimental autoimmune encephalomyelitis (EAE)," Experimental Neurology, vol. 241, pp. 113-121, 2013.

[54] L. H. Guo and H. J. Schluesener, "Lesional accumulation of $\mathrm{P} 2 \mathrm{X} 4$ receptor+ macrophages in rat CNS during experimental autoimmune encephalomyelitis," Neuroscience, vol. 134, no. 1 , pp. 199-205, 2005.

[55] M. Tsuda, Y. Shigemoto-Mogami, S. Koizumi et al., "P2X4 receptors induced in spinal microglia gate tactile allodynia after nerve injury," Nature, vol. 424, no. 6950, pp. 778-783, 2003.

[56] H. Kim, C. Moon, M. Ahn et al., "Increased phosphorylation of cyclic AMP response element-binding protein in the spinal cord of Lewis rats with experimental autoimmune encephalomyelitis," Brain Research, vol. 1162, no. 1, pp. 113-120, 2007.

[57] A. R. Glabinski, M. Tani, V. K. Tuohy, R. J. Tuthill, and R. M. Ransohoff, "Central nervous system chemokine mRNA accumulation follows initial leukocyte entry at the onset of acute murine experimental autoimmune encephalomyelitis," Brain, Behavior, and Immunity, vol. 9, no. 4, pp. 315-330, 1995.

[58] D. J. Mahad and R. M. Ransohoff, “The role of MCP-1 (CCL2) and CCR2 in multiple sclerosis and experimental autoimmune encephalomyelitis (EAE)," Seminars in Immunology, vol. 15, no. 1, pp. 23-32, 2003.

[59] C. Abbadie, J. A. Lindia, A. M. Cumiskey et al., "Impaired neuropathic pain responses in mice lacking the chemokine receptor CCR2," Proceedings of the National Academy of Sciences of the United States of America, vol. 100, no. 13, pp. 7947-7952, 2003.

[60] B. T. Fife, G. B. Huffnagle, W. A. Kuziel, and W. J. Karpus, "CC chemokine receptor 2 is critical for induction of experimental autoimmune encephalomyelitis," Journal of Experimental Medicine, vol. 192, no. 6, pp. 899-905, 2000.

[61] S. Bhangoo, D. Ren, R. J. Miller et al., "Delayed functional expression of neuronal chemokine receptors following focal nerve demyelination in the rat: a mechanism for the development of chronic sensitization of peripheral nociceptors," Molecular Pain, vol. 3, article 38, 2007.

[62] P. Wipfler, A. Harrer, G. Pilz, K. Oppermann, E. Trinka, and J. Kraus, "Recent developments in approved and oral multiple sclerosis treatment and an update on future treatment options," Drug Discovery Today, vol. 16, no. 1-2, pp. 8-21, 2011.

[63] O. Hadjimichael, R. D. Kerns, M. A. Rizzo, G. Cutter, and T. Vollmer, "Persistent pain and uncomfortable sensations in persons with multiple sclerosis," Pain, vol. 127, no. 1-2, pp. 3541, 2007.
[64] D. Seixas, V. Galhardo, M. J. Sá, J. Guimarães, and D. Lima, "Pain in portuguese patients with multiple sclerosis," Frontiers in Neurology, vol. 2, article 20, 2011.

[65] N. C. Cutter, D. D. Scott, J. C. Johnson, and G. Whiteneck, "Gabapentin effect on spasticity in multiple sclerosis: a placebocontrolled, randomized trial," Archives of Physical Medicine and Rehabilitation, vol. 81, no. 2, pp. 164-169, 2000.

[66] M. E. Mueller, M. Gruenthal, W. L. Olson, and W. H. Olson, "Gabapentin for relief of upper motor neuron symptoms in multiple sclerosis," Archives of Physical Medicine and Rehabilitation, vol. 78, no. 5, pp. 521-524, 1997.

[67] L. M. Samkoff, M. Daras, A. J. Tuchman, and B. S. Koppel, "Amelioration of refractory dysesthetic limb pain in multiple sclerosis by gabapentin," Neurology, vol. 49, no. 1, pp. 304-305, 1997.

[68] C. Solaro, G. L. Lunardi, E. Capello et al., "An open-label trial of gabapentin treatment of paroxysmal symptoms in multiple sclerosis patients," Neurology, vol. 51, no. 2, pp. 609-611, 1998.

[69] C. O. Asante, V. C. Wallace, and A. H. Dickenson, "Mammalian target of rapamycin signaling in the spinal cord is required for neuronal plasticity and behavioral hypersensitivity associated with neuropathy in the rat," Journal of Pain, vol. 11, no. 12, pp. 1356-1367, 2010.

[70] E. Norsted Gregory, S. Codeluppi, J. A. Gregory, J. Steinauer, and C. I. Svensson, "Mammalian target of rapamycin in spinal cord neurons mediates hypersensitivity induced by peripheral inflammation," Neuroscience, vol. 169, no. 3, pp. 1392-1402, 2010.

[71] S. M. Géranton, L. Jiménez-Díaz, C. Torsney et al., "A rapamycin-sensitive signaling pathway is essential for the full expression of persistent pain states," Journal of Neuroscience, vol. 29, no. 47, pp. 15017-15027, 2009.

[72] M. Esposito, F. Ruffini, M. Bellone et al., "Rapamycin inhibits relapsing experimental autoimmune encephalomyelitis by both effector and regulatory T cells modulation," Journal of Neuroimmunology, vol. 220, no. 1-2, pp. 52-63, 2010.

[73] H. Kusaba, P. Ghosh, R. Derin et al., "Interleukin-12-induced interferon- $\gamma$ production by human peripheral blood $\mathrm{T}$ cells is regulated by mammalian target of rapamycin (mTOR)," Journal of Biological Chemistry, vol. 280, no. 2, pp. 1037-1043, 2005.

[74] J. F. Stover, U. E. Pleinesf, M. C. Morganti-Kossmannf, T. Kossmannf, K. Lowitzschj, and O. S. Kempski, "Neurotransmitters in cerebrospinal fluid reflect pathological activity," European Journal of Clinical Investigation, vol. 27, no. 12, pp. 1038-1043, 1997.

[75] R. Srinivasan, N. Sailasuta, R. Hurd, S. Nelson, and D. Pelletier, "Evidence of elevated glutamate in multiple sclerosis using magnetic resonance spectroscopy at 3 T," Brain, vol. 128, no. 5, pp. 1016-1025, 2005.

[76] M. Vercellino, A. Merola, C. Piacentino et al., "Altered glutamate reuptake in relapsing-remitting and secondary progressive multiple sclerosis cortex: correlation with microglia infiltration, demyelination, and neuronal and synaptic damage," Journal of Neuropathology and Experimental Neurology, vol. 66, no. 8, pp. 732-739, 2007.

[77] P. Werner, D. Pitt, and C. S. Raine, "Multiple sclerosis: altered glutamate homeostasis in lesions correlates with oligodendrocyre and axonal damage," Annals of Neurology, vol. 50, no. 2, pp. 169-180, 2001.

[78] A. Vallejo-Illarramendi, M. Domercq, F. Pérez-Cerdá, R. Ravid, and C. Matute, "Increased expression and function of glutamate transporters in multiple sclerosis," Neurobiology of Disease, vol. 21, no. 1, pp. 154-164, 2006. 
[79] D. Piani, K. Frei, K. Q. Do, M. Cuenod, and A. Fontana, "Murine brain macrophages induce NMDA receptor mediated neurotoxicity in vitro by secreting glutamate," Neuroscience Letters, vol. 133, no. 2, pp. 159-162, 1991.

[80] S. K. Garg, R. Banerjee, and J. Kipnis, "Neuroprotective immunity: T cell-derived glutamate endows astrocytes with a neuroprotective phenotype," Journal of Immunology, vol. 180, no. 6, pp. 3866-3873, 2008.

[81] S. M. Fine, R. A. Angel, S. W. Perry et al., "Tumor necrosis factor $\alpha$ inhibits glutamate uptake by primary human astrocytes. Implications for pathogenesis of HIV-1 dementia," Journal of Biological Chemistry, vol. 271, no. 26, pp. 15303-15306, 1996.

[82] T. Smith, A. Groom, B. Zhu, and L. Tukski, "Autoimmune encephalomyelitis ameliorated by AMPA antagonists," Nature Medicine, vol. 6, no. 1, pp. 62-66, 2000.

[83] A. S. Basso, D. Frenkel, F. J. Quintana et al., "Reversal of axonal loss and disability in a mouse model of progressive multiple sclerosis," Journal of Clinical Investigation, vol. 118, no. 4, pp. 1532-1543, 2008.

[84] M. Ohgoh, T. Hanada, T. Smith et al., "Altered expression of glutamate transporters in experimental autoimmune encephalomyelitis," Journal of Neuroimmunology, vol. 125, no. 1-2, pp. 170-178, 2002.

[85] N. Melzer, S. G. Meuth, D. Torres-Salazar et al., "A $\beta$-lactam antibiotic dampens excitotoxic inflammatory CNS damage in a mouse model of multiple sclerosis," PLoS ONE, vol. 3, no. 9, Article ID e3149, 2008.

[86] G. Comi, D. Jeffery, L. Kappos et al., "Placebo-controlled trial of oral laquinimod for multiple sclerosis," The New England Journal of Medicine, vol. 366, pp. 1000-1009, 2012.

[87] C. Ford, A. D. Goodman, K. Johnson et al., "Continuous long-term immunomodulatory therapy in relapsing multiple sclerosis: results from the 15 -year analysis of the US prospective open-label study of glatiramer acetate," Multiple Sclerosis, vol. 16, no. 3, pp. 342-350, 2010.

[88] B. Bielekova, N. Richert, T. Howard et al., "Humanized antiCD25 (daclizumab) inhibits disease activity in multiple sclerosis patients failing to respond to interferon $\beta$," Proceedings of the National Academy of Sciences of the United States of America, vol. 101, no. 23, pp. 8705-8708, 2004.

[89] B. C. Kieseier, "The mechanism of action of interferon- $\beta$ in relapsing multiple sclerosis," CNS Drugs, vol. 25, no. 6, pp. 491502, 2011.

[90] V. Brinkmann, M. D. Davis, C. E. Heise et al., "The immune modulator FTY720 targets sphingosine 1-phosphate receptors," Journal of Biological Chemistry, vol. 277, no. 24, pp. 21453-21457, 2002.

[91] M. Webb, C. S. Tham, F. F. Lin et al., "Sphingosine 1-phosphate receptor agonists attenuate relapsing-remitting experimental autoimmune encephalitis in SJL mice," Journal of Neuroimmunology, vol. 153, no. 1-2, pp. 108-121, 2004.

[92] M. Fujino, N. Funeshima, Y. Kitazawa et al., "Amelioration of experimental autoimmune encephalomyelitis in Lewis rats by FTY720 treatment," Journal of Pharmacology and Experimental Therapeutics, vol. 305, no. 1, pp. 70-77, 2003.

[93] H. Kataoka, K. Sugahara, K. Shimano et al., "FTY720, sphingosine 1-phosphate receptor modulator, ameliorates experimental autoimmune encephalomyelitis by inhibition of $\mathrm{T}$ cell infiltration," Cellular \& Molecular Immunology, vol. 2, no. 6, pp. 439448, 2005.

[94] D. Papadopoulos, J. Rundle, R. Patel et al., "FTY720 ameliorates MOG-induced experimental autoimmune encephalomyelitis by suppressing both cellular and humoral immune responses," Journal of Neuroscience Research, vol. 88, no. 2, pp. 346-359, 2010.

[95] M. Rausch, P. Hiestand, C. A. Foster, D. R. Baumann, C. Cannet, and M. Rudin, "Predictability of FTY720 efficacy in experimental autoimmune encephalomyelitis by in vivo macrophage tracking: clinical implications for ultrasmall superparamagnetic iron oxide-enhanced magnetic resonance imaging," Journal of Magnetic Resonance Imaging, vol. 20, no. 1, pp. 16-24, 2004.

[96] P. J. Gonzalez-Cabrera, S. M. Cahalan, N. Nguyen et al., "S1P(1) receptor modulation with cyclical recovery from lymphopenia ameliorates mouse model of multiple sclerosis," Molecular Pharmacology, vol. 81, pp. 166-174, 2012.

[97] J. W. Choi, S. E. Gardell, D. R. Herr et al., "FTY720 (fingolimod) efficacy in an animal model of multiple sclerosis requires astrocyte sphingosine 1-phosphate receptor 1 (S1P1) modulation," Proceedings of the National Academy of Sciences of the United States of America, vol. 108, no. 2, pp. 751-756, 2011.

[98] V. E. Miron, A. Schubart, and J. P. Antel, "Central nervous system-directed effects of FTY720 (fingolimod)," Journal of the Neurological Sciences, vol. 274, no. 1-2, pp. 13-17, 2008.

[99] S. Rossi, T. Lo Giudice, V. De Chiara et al., "Oral fingolimod rescues the functional deficits of synapses in experimental autoimmune encephalomyelitis," British Journal of Pharmacology, vol. 165, pp. 861-869, 2012.

[100] C. A. Foster, L. M. Howard, A. Schweitzer et al., "Brain penetration of the oral immunomodulatory drug FTY720 and its phosphorylation in the central nervous system during experimental autoimmune encephalomyelitis: consequences for mode of action in multiple sclerosis," Journal of Pharmacology and Experimental Therapeutics, vol. 323, no. 2, pp. 469-476, 2007.

[101] L. Kappos, J. Antel, G. Comi et al., "Oral fingolimod (FTY720) for relapsing multiple sclerosis," The New England Journal of Medicine, vol. 355, no. 11, pp. 1124-1140, 2006.

[102] L. Kappos, E. W. Radue, P. O’Connor et al., "A placebocontrolled trial of oral fingolimod in relapsing multiple sclerosis," The New England Journal of Medicine, vol. 362, no. 5, pp. 387-401, 2010.

[103] J. A. Cohen, F. Barkhof, G. Comi et al., "Oral fingolimod or intramuscular interferon for relapsing multiple sclerosis," The New England Journal of Medicine, vol. 362, no. 5, pp. 402-415, 2010.

[104] B. Balatoni, M. K. Storch, E. M. Swoboda et al., "FTY720 sustains and restores neuronal function in the DA rat model of MOG-induced experimental autoimmune encephalomyelitis," Brain Research Bulletin, vol. 74, no. 5, pp. 307-316, 2007.

[105] O. Coste, S. Pierre, C. Marian et al., "Antinociceptive activity of the S1P-receptor agonist FTY720," Journal of Cellular and Molecular Medicine, vol. 12, no. 3, pp. 995-1004, 2008.

[106] R. A. Hughes and D. R. Cornblath, "Guillain-Barré syndrome," The Lancet, vol. 366, no. 9497, pp. 1653-1666, 2005.

[107] N. Yuki and H. P. Hartung, "Guillain-Barre syndrome," The New England Journal of Medicine, vol. 366, pp. 2294-2304, 2012.

[108] R. A. C. Hughes, R. D. M. Hadden, N. A. Gregson, and K. J. Smith, "Pathogenesis of Guillain-Barre syndrome," Journal of Neuroimmunology, vol. 100, no. 1-2, pp. 74-97, 1999.

[109] D. E. Moulin, N. Hagen, T. E. Feasby, R. Amireh, and A. Hahn, "Pain in Guillain-Barre syndrome," Neurology, vol. 48, no. 2, pp. 328-331, 1997.

[110] A. F. Hahn, "Experimental allergic neuritis (EAN) as a model for the immune-mediated demyelinating neuropathies," Revue Neurologique, vol. 152, no. 5, pp. 328-332, 1996. 
[111] H. C. Shin, E. F. McFarlane, J. D. Pollard, and E. G. S. Watson, "Induction of experimental allergic neuritis with synthetic peptides from myelin P2 protein," Neuroscience Letters, vol. 102, no. 2-3, pp. 309-312, 1989.

[112] C. M. Gabriel, R. A. C. Hughes, S. E. Moore, K. J. Smith, and F. S. Walsh, "Induction of experimental autoimmune neuritis with peripheral myelin protein-22," Brain, vol. 121, no. 10, pp. 18951902, 1998.

[113] V. Martinez, D. Fletcher, F. Martin et al., "Small fibre impairment predicts neuropathic pain in Guillain-Barre syndrome," Pain, vol. 151, pp. 53-60, 2010.

[114] C. L. Pan, T. J. Tseng, Y. H. Lin, M. C. Chiang, W. M. Lin, and S. T. Hsieh, "Cutaneous innervation in Guillain-Barré syndrome: pathology and clinical correlations," Brain, vol. 126, no. 2, pp. 386-397, 2003.

[115] G. Moalem-Taylor, H. N. Allbutt, M. D. Iordanova, and D. J. Tracey, "Pain hypersensitivity in rats with experimental autoimmune neuritis, an animal model of human inflammatory demyelinating neuropathy," Brain, Behavior, and Immunity, vol. 21, no. 5, pp. 699-710, 2007.

[116] Z. Zhang, Z. Y. Zhang, U. Fauser, and H. J. Schluesener, "Mechanical allodynia and spinal up-regulation of P2X4 receptor in experimental autoimmune neuritis rats," Neuroscience, vol. 152, no. 2, pp. 495-501, 2008.

[117] Z. Y. Zhang, Z. Zhang, U. Fauser, and H. J. Schluesener, "Improved outcome of EAN, an animal model of GBS, through amelioration of peripheral and central inflammation by minocycline," Journal of Cellular and Molecular Medicine, vol. 13, no. 2, pp. 341-351, 2009.

[118] Z. Zhang, Z. Y. Zhang, and H. J. Schluesener, "Compound A, a plant origin ligand of glucocorticoid receptors, increases regulatory T cells and $\mathrm{M} 2$ macrophages to attenuate experimental autoimmune neuritis with reduced side effects," Journal of Immunology, vol. 183, no. 5, pp. 3081-3091, 2009.

[119] P. J. Austin, C. F. Kim, C. J. Perera, and G. Moalem-Taylor, "Regulatory $\mathrm{T}$ cells attenuate neuropathic pain following peripheral nerve injury and experimental autoimmune neuritis," Pain, vol. 153, pp. 1916-1931, 2012.

[120] L. Luongo, M. Sajic, J. Grist, A. K. Clark, S. Maione, and M. Malcangio, "Spinal changes associated with mechanical hypersensitivity in a model of Guillain-Barré syndrome," Neuroscience Letters, vol. 437, no. 2, pp. 98-102, 2008.

[121] P. A. van Doorn, L. Ruts, and B. C. Jacobs, "Clinical features, pathogenesis, and treatment of Guillain-Barré syndrome," The Lancet Neurology, vol. 7, no. 10, pp. 939-950, 2008.

[122] L. Ruts, P. A. van Doorn, R. Lombardi et al., "Unmyelinated and myelinated skin nerve damage in Guillain-Barre syndrome: correlation with pain and recovery," Pain, vol. 153, pp. 399-409, 2012.

[123] S. Jander and G. Stoll, "Interleukin-18 is induced in acute inflammatory demyelinating polyneuropathy," Journal of Neuroimmunology, vol. 114, no. 1-2, pp. 253-258, 2001.

[124] C. L. R. De La Hoz, F. R. Castro, L. M. B. Santos, and F. Langone, "Distribution of inducible nitric oxide synthase and tumor necrosis factor- $\alpha$ in the peripheral nervous system of lewis rats during ascending paresis and spontaneous recovery from experimental autoimmune neuritis," NeuroImmunoModulation, vol. 17, no. 1, pp. 56-66, 2009.

[125] T. Trang, S. Beggs, and M. W. Salter, "Brain-derived neurotrophic factor from microglia: a molecular substrate for neuropathic pain," Neuron Glia Biology, vol. 7, pp. 99-108, 2011.
[126] S. Kastenbauer, U. Koedel, M. Wick, B. C. Kieseier, H. P. Hartung, and H. W. Pfister, "CSF and serum levels of soluble fractalkine (CX3CL1) in inflammatory diseases of the nervous system," Journal of Neuroimmunology, vol. 137, no. 1-2, pp. 210 217,2003

[127] X. L. Li, Y. C. Dou, Y. Liu et al., "Atorvastatin ameliorates experimental autoimmune neuritis by decreased Th1/Th17 cytokines and up-regulated T regulatory cells," Cellular Immunology, vol. 271, pp. 455-461, 2011.

[128] X. Q. Shi, T. K. Y. Lim, S. Lee, Y. Q. Zhao, and J. Zhang, "Statins alleviate experimental nerve injury-induced neuropathic pain," Pain, vol. 152, no. 5, pp. 1033-1043, 2011.

[129] F. Giuliani, W. Hader, and V. W. Yong, "Minocycline attenuates $\mathrm{T}$ cell and microglia activity to impair cytokine production in T cell-microglia interaction," Journal of Leukocyte Biology, vol. 78, no. 1, pp. 135-143, 2005.

[130] P. M. Hughes, G. M. A. Wells, J. M. Clements et al., "Matrix metalloproteinase expression during experimental autoimmune neuritis," Brain, vol. 121, no. 3, pp. 481-494, 1998.

[131] B. C. Kieseier, J. M. Clements, H. B. Pischel et al., "Matrix metalloproteinases MMP-9 and MMP-7 are expressed in experimental autoimmune neuritis and the Guillain-Barre syndrome," Annals of Neurology, vol. 43, no. 4, pp. 427-434, 1998.

[132] C. J. Klein, V. A. Lennon, P. A. Aston, A. McKeon, and S. J. Pittock, "Chronic pain as a manifestation of potassium channelcomplex autoimmunity," Neurology, vol. 79, pp. 1136-1144, 2012. 


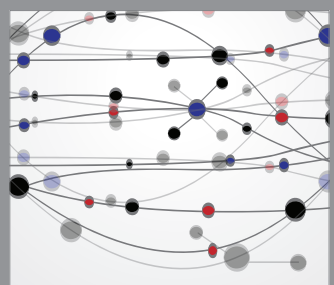

The Scientific World Journal
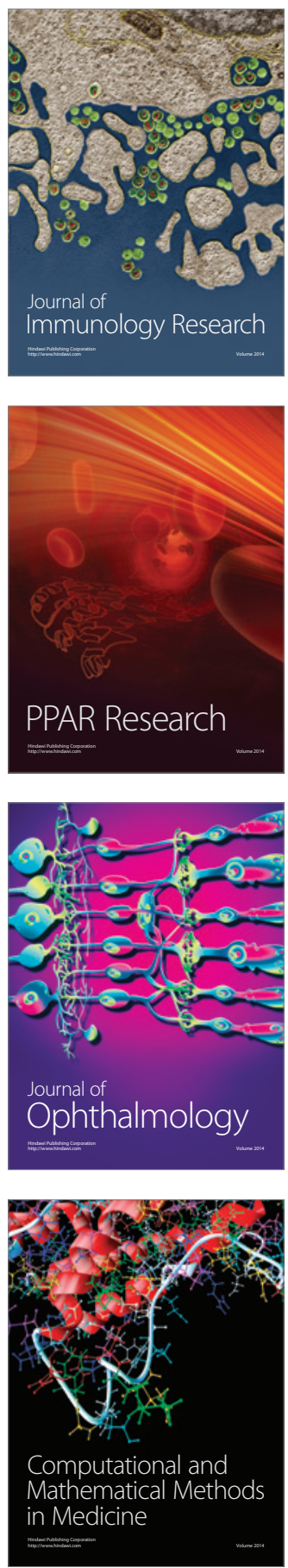

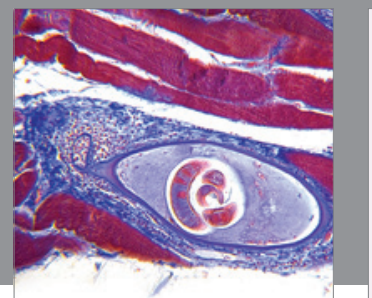

Gastroenterology

Research and Practice
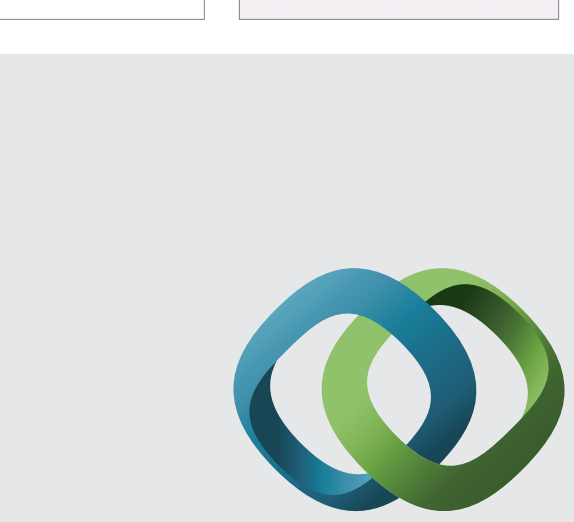

\section{Hindawi}

Submit your manuscripts at

http://www.hindawi.com
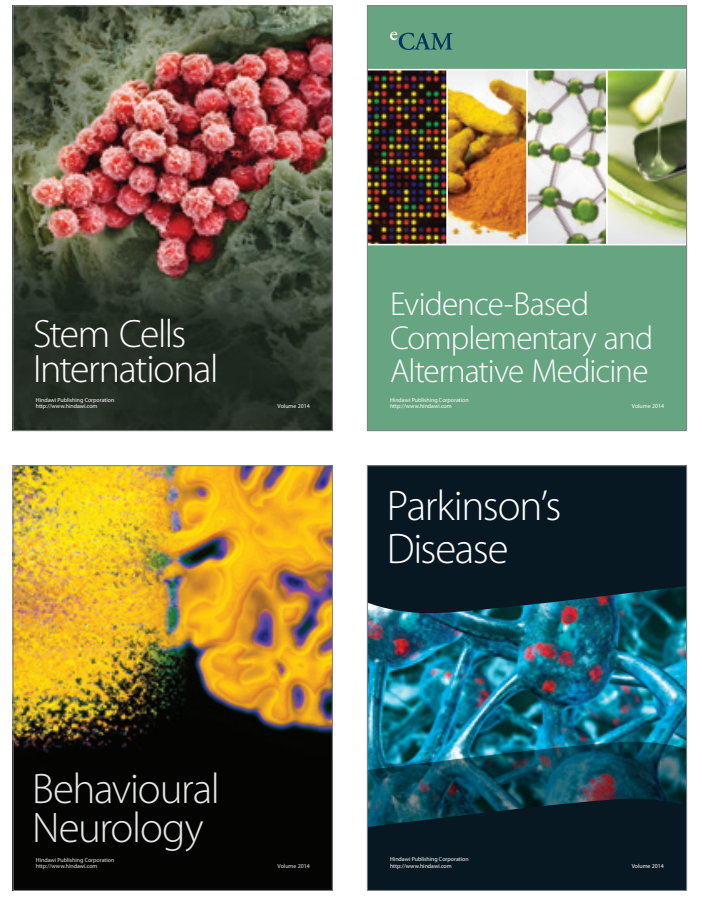
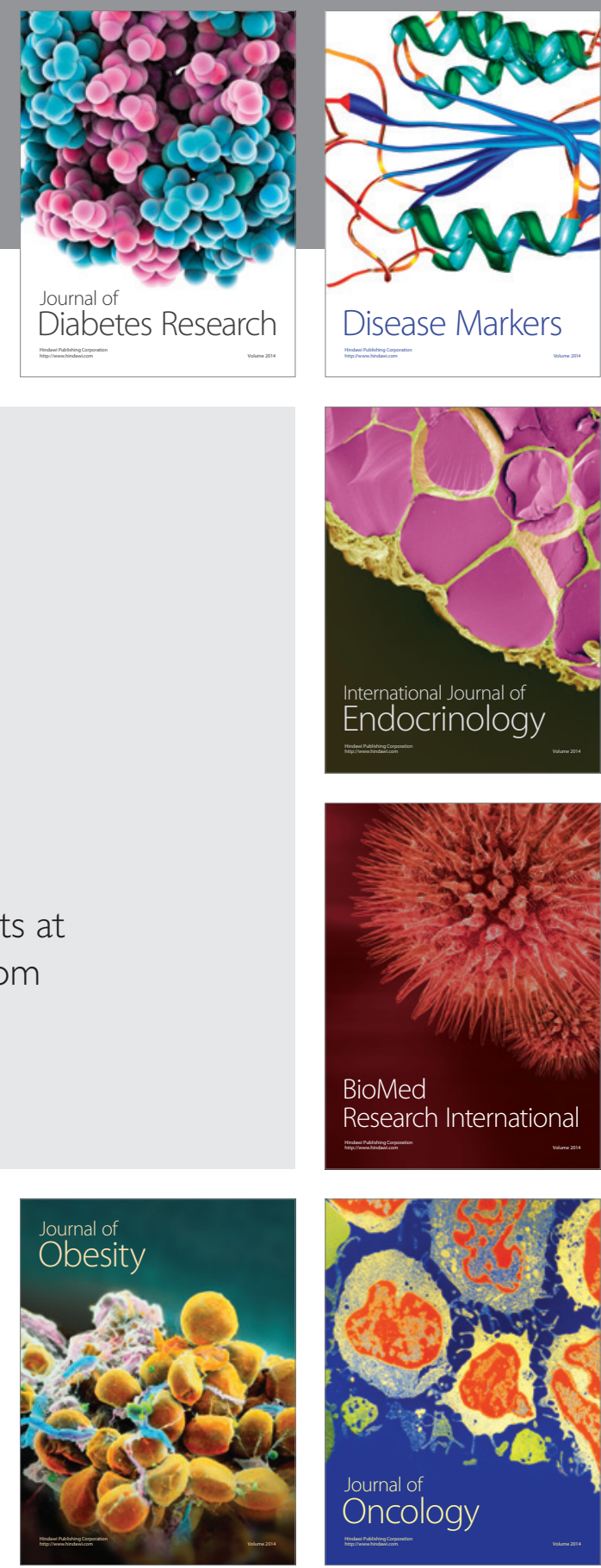

Disease Markers
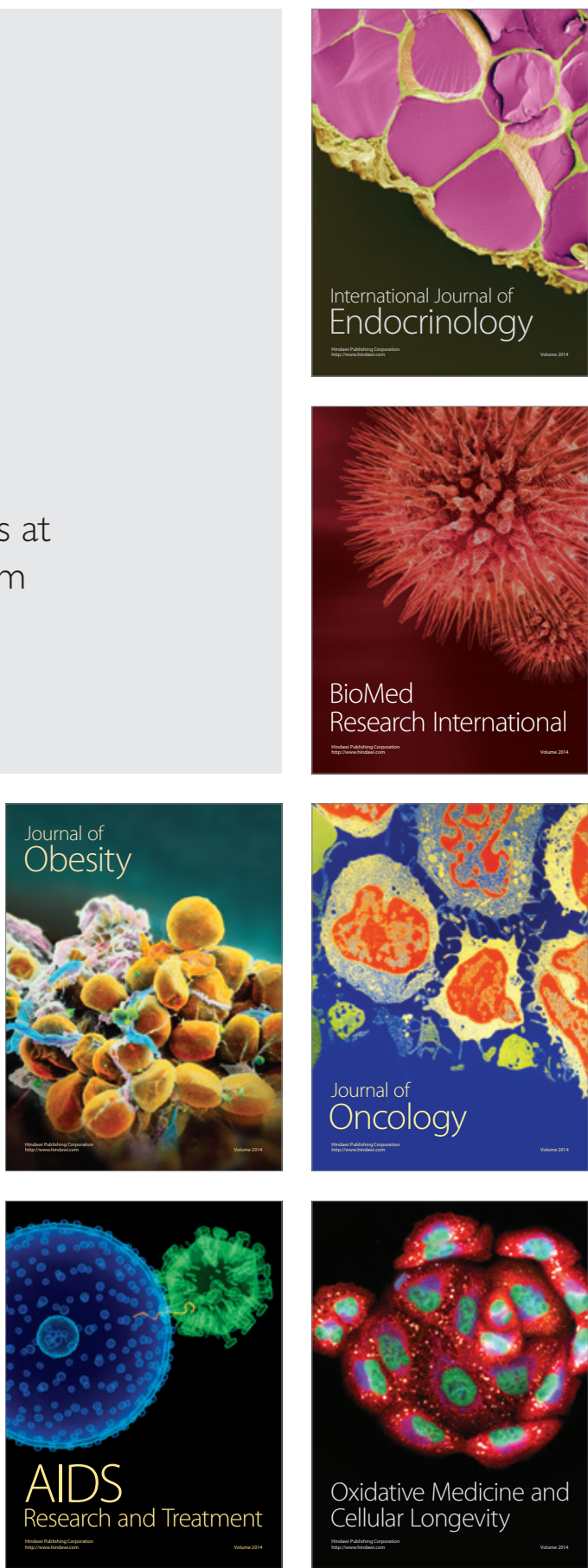